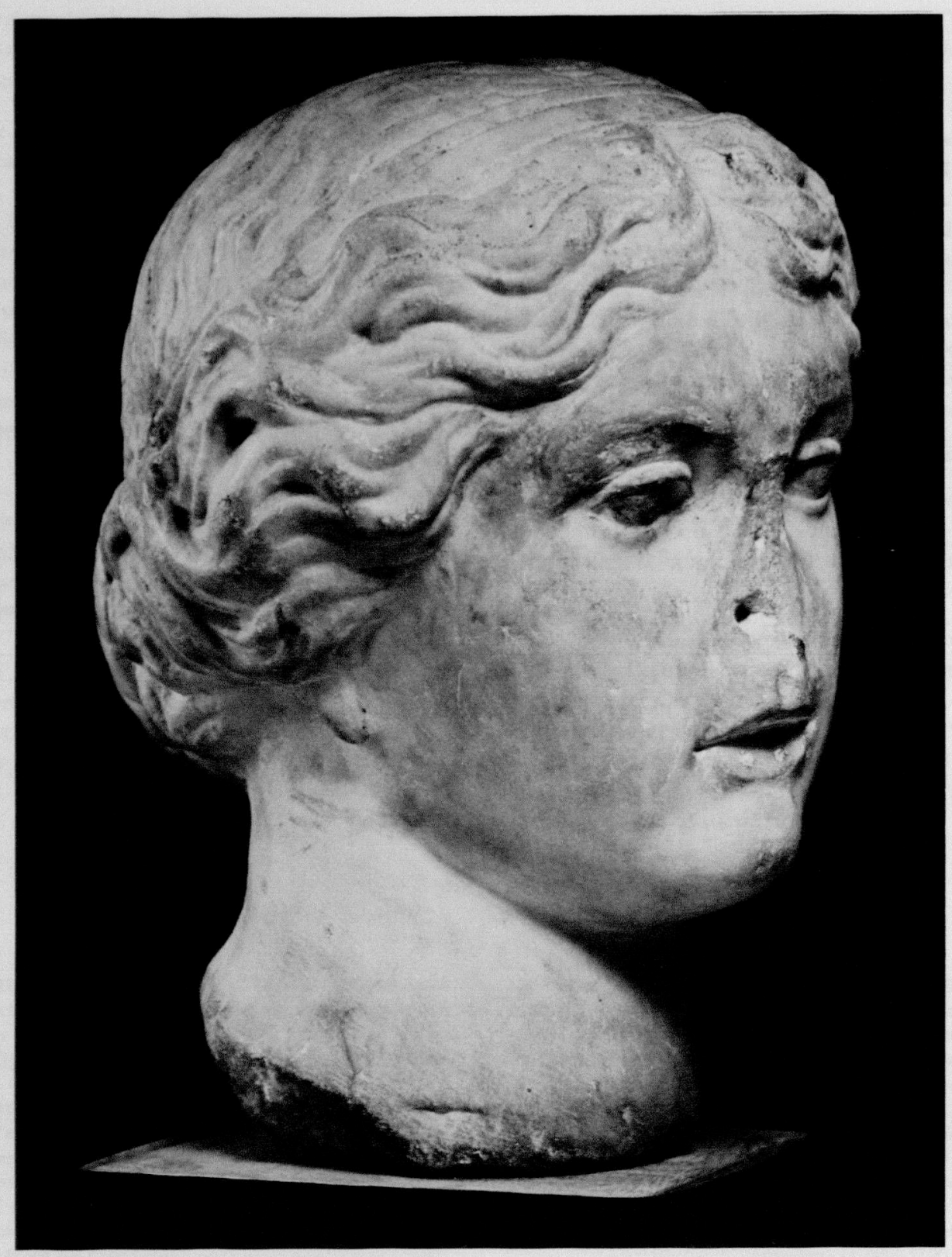

Fig. 1 : Portrait de femme

Musée royal de Mariemont (Photo du Musée) 


\section{UN PORTRAIT FÉMININ IDÉALISÉ D’ÉPOQUE ROMAINE AU MUSÉE DE MARIEMONT ( $\left.{ }^{*}\right)$}

\begin{abstract}
Alors que j'étudiais un portrait de femme très idéalisé dans une collection privée de Münster $\left({ }^{1}\right)$, j'eus l'attention attirée, à l'occasion d'une visite au Musée de Mariemont en 1980, par une tête féminine qui présente également des traits idéalisés (fig. 1-3, 7-8). Toutefois, son cas allait se révéler à l'examen quelque peu différent de celui du portrait de Münster. La tête de Mariemont aurait été trouvée aux environs de Maastricht (Pays-Bas). On ne possède aucune autre précision sur les circonstances et la date de sa découverte. On sait seulement qu'elle a fait partie de la collection du Professeur J. Brassine de Liège. Sur la base des renseignements donnés par ce dernier, Espérandieu la publia en 1928 dans son Recueil en tant que trouvaille archéologique, avec deux illustrations médiocres $\left(^{2}\right)$. En 1956, elle entrait, avec le reste de la collection Brassine, au Musée de Mariemont, où elle est actuellement exposée $\left({ }^{3}\right)$.
\end{abstract}

La tête, en marbre blanc jaunâtre, est conservée sur une hauteur de $32 \mathrm{~cm}$. La tête proprement dite, du menton au sommet du crâne, mesure $25 \mathrm{~cm}$. Le nez et les lèvres, qui devaient être fortement endommagés. ont été coupés net par une restauration moderne au

(*) Je remercie chaleureusement M. G. Donnay, directeur du Musée. pour les informations qu'il m'a fournies sur la pièce et. surtout, pour son invitation à publier ici les réflexions qu elle $\mathrm{m}^{*} \mathrm{a}$ inspirées, ainsi que pour la traduction de mon texte.

(1) R. Stupperich, Boreas, 4, 1981, p. 201-206, pl. 31-32.

$\left.{ }^{2}{ }^{2}\right)$ E. ESPERANDIEU, Recueil général des has-reliefs, statues tournant du siècle $\left({ }^{4}\right)$. Les compléments, fixés alors à l'aide de tenons, ont été ensuite retirés et ce, comme on peut le voir sur les illustrations, dès avant la publication d'Espérandieu. Au moment de l'acquisition de la tête par le Musée de Mariemont, le nez avait été à nouveau refait en plâtre; cette restauration a été enlevée en 1966. Pour juger de la qualité de l'œuvre, il faut tenir compte de l'impression défavorable produite par ces horribles mutilations modernes. Elle est également enlaidie par de nombreuses griffes et éclats superficiels, en particulier dans la région des sourcils l'arcade sourcilière droite a été restaurée en plâtre jusqu'à la paupière supérieure -, ainsi que par un nettoyage trop poussé, qui a laissé des traces très nettes, notamment sur certaines parties des chairs. Le fait que le travail de la couronne de cheveux apparait moins précis et moins accentué que, par exemple, celui du chignon semble, en revanche, devoir être attribué, plutôt qu'au nettoyage moderne, à une différence fondamentale de style à l'intérieur même de la coiffure.

Une cavité rectangulaire de 2.5 à $3 \mathrm{~cm}$ sur $9 \mathrm{~cm}$ environ, qui présente un creux de $4 \mathrm{~cm}$ à la partie supérieure, a été ménagée à l'arrière du crâne. peut-

et bustes de la Gaule romaine. X (Paris. 1928), n" 7528

(3) Inv. Ac.622.B. G. Donnay, dans Rome, ses origines et son Empire. Catalogue (Musée de Mariemont. 1966). p. 67. $\mathrm{n}^{\prime \prime} 166$ (identification de J. Ch. Balty): STupperich. art. cité (cidessus n. 11. p. 205-206. n. 6.

(1) $C^{2}$ est la date suggérée notamment par ESPERANDIFt. op. cit. 


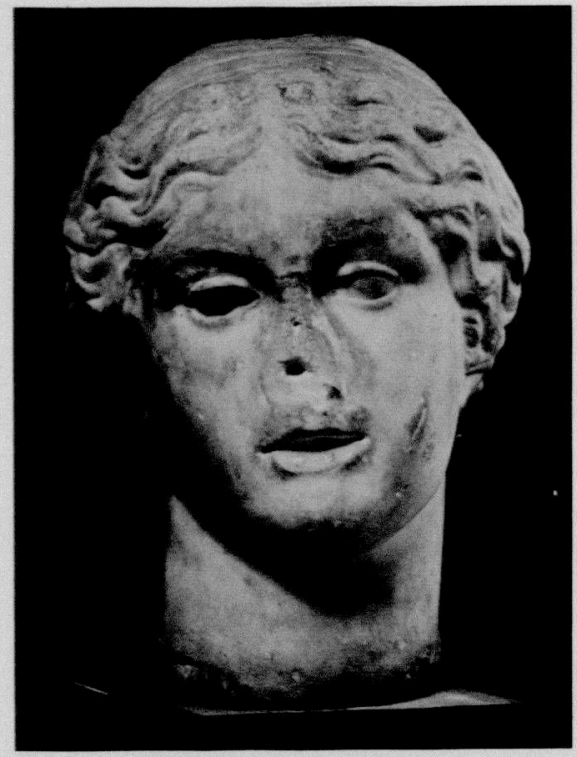

Fig. 2 : Portrait de femme. face

Musée royal de Mariemont (Photo du Musée)

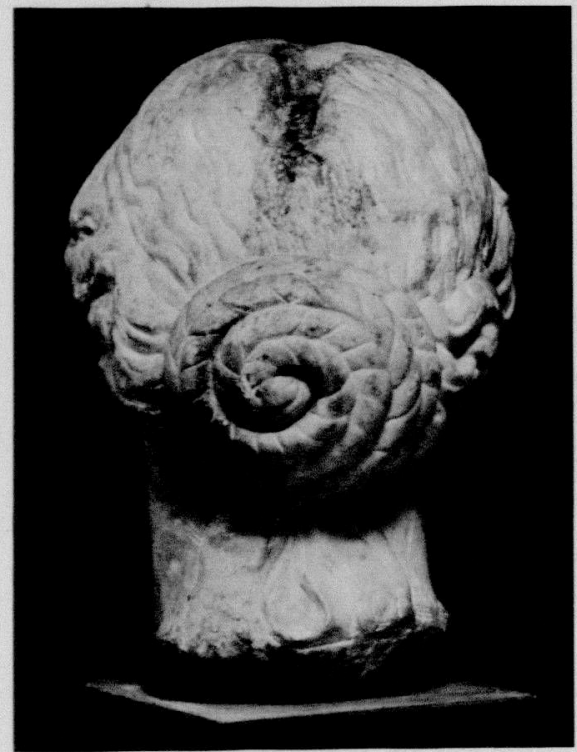

Fig. 3 : Portrait de femme, dos

Musée royal de Mariemont (Photo du Musée)

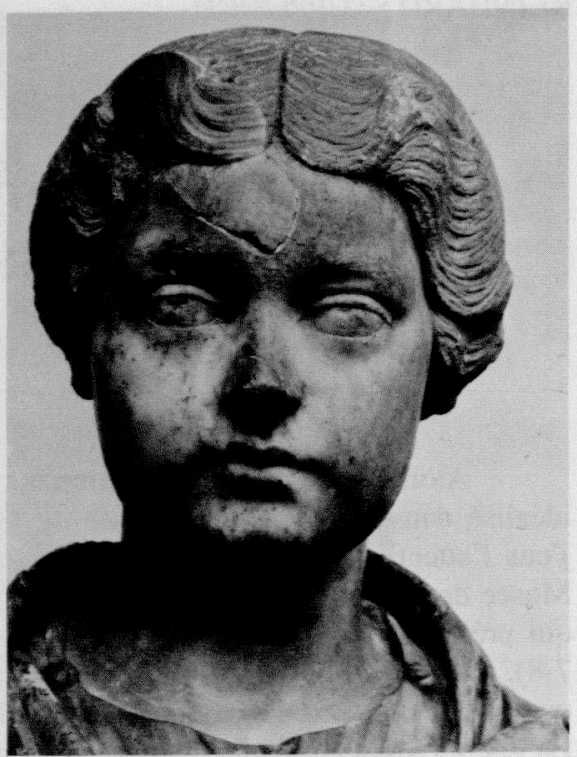

Fig. 4 : Portrait de Lucilla, face Londres, British Museum, Inv. 1912 (Photo British Museum)

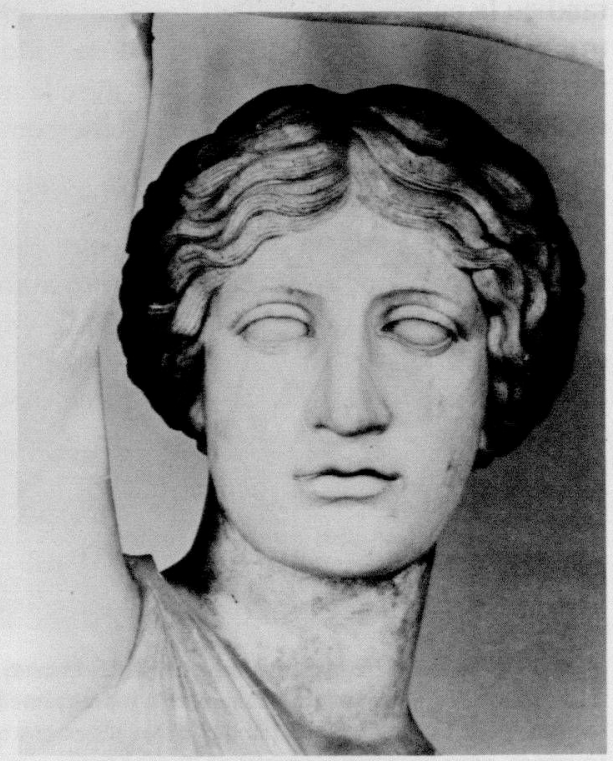

Fig. 5 : Tête de l'Amazone de Sosiklès, face Rome, Musée du Capitole

(Photo Deutsches archäologisches Institut, Rome) 
être pour recevoir une fixation moderne $\left(^{5}\right)$. L'extrémité inférieure du cou, plus large, présente un piquetage antique grossier, qui la destine clairement à être encastrée. À l'avant, le bord antique est brisé, mais il ne pouvait descendre beaucoup plus bas. Cette observation plaide en faveur de l'appartenance de la tête à une statue plutôt qu'à un buste et indique, en outre, que cette statue devait porter un vêtement qui montait relativement haut sur le cou.

Le visage est ovale, les joues sont légèrement pleines, le menton est petit et rond. Sous les arcades sourcilières - en fait, très abîmées - qui s'arrondis-

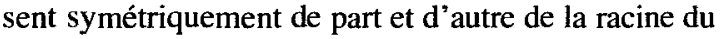
nez, des yeux relativement grands aux paupières étroites se détachent du creux d'orbites légèrement arquées à la partie supérieure. L'iris et la pupille ne sont pas indiqués. Il n'y a pas grand-chose à dire du nez; aucune ride n'est visible sur les joues autour du nez et de la bouche; celle-ci, petite, a disparu jusqu'aux trous de foret qui marquent la commissure des lèvres.

Une couronne de cheveux encadre le front; une raie médiane la divise en deux bandeaux de mèches bouclées qui se chevauchent partiellement, passent sur les oreilles, dont seul le lobe est visible, et disparaissent à l'arrière dans un chignon. Les boucles, tantôt larges, tantôt étroites, les creux et les saillies, les limites supérieure et inférieure ont été taillés uniquement au ciseau, sans recours au foret. Un ruban, orné d'un galon gravé, est posé au-dessus de la couronne de cheveux, dont les boucles le recouvrent en partie; il est noué directement au-dessus du chignon et ses larges bouts tombent sur la nuque. Ce chignon est relativement petit, posé plutôt bas et formé simplement d'une

(5) En raison de l'emplacement de ce creux, on pourrait penser, par exemple, à l'encastrement d'une partie rapportée de la chevelure, mais le procédé serait sans parallèle et quelque peu aberrant. $G$. Donnay considère le travail comme moderne.

(") Ci-dessus n. 2.

(7) Voir, p. ex. H. von Heintz, Die antiken Porträts im Schlop Fasanerie bei Fulda (Mayence, 1968), n" 14, pl. 23 et 110. $\mathrm{c}$-d; Fr. PUULSEN, Porträtstudien in norditalienischen Provinamu- seule grosse natte, tressée autour d'une mèche centrale continue, enroulée en spirale vers la gauche. Pour le reste, la chevelure est légèrement ondulée sur le crâne et fortement tirée vers l'arrière - ou, sur le côté droit, vers l'arrière-droit — afin d'être prise dans la natte qui s'amorce à cet endroit.

Le cou assez fort montre une légère torsion de la tête vers la droite, qui est également responsable de deux légères rides sur la partie droite du cou.

On remarque dans cette tête une opposition entre des composantes de style différent, ce qui lui confère un intérêt particulier. Lą symétrie du visage aux traits juvéniles, le flot élégant des boucles qui le couronnent, l'absence de caractères individuels rappellent les représentations idéalisées des dieux dans l'art classique. Il est, dès lors, compréhensible qu'Espérandieu l'ait rangée parmi les images de Vénus. Il la tient pour une œuvre gréco-romaine, c'est-à-dire sans doute pour une copie ou une variante d'un modèle grec classique, et la date du $1^{\text {er }}$ siècle après J.-C. $\left(^{6}\right)$. D'un autre côté, on ne peut nier qu'il s'agit d'un portrait; la rondeur des joues, la forme du menton sont, par exemple, impensables pour une image divine classique ou classicisante. Le chignon surtout ne saurait appartenir à une telle image, mais à une coiffure féminine d'époque romaine.

De petits chignons de ce type apparaissent occasionnellement à la fin de la République et au début du règne d'Auguste $\left({ }^{7}\right)$, parfois combinés avec une natte ramenée dans l'axe du crâne, ensuite surtout à l'époque antonine sous différentes formes, dans des portraits de Faustine la Jeune, de Lucilla et de Crispina seen (Copenhague, 1928), p. 23-24, no 3. fig. 41-42: V. POULSEN, Les portraits romains (Copenhague, 1962), $\mathrm{n}^{0}$ 100, pl. 176: B.M. FELETTI MAs. Museo Nazionale Romano. I ritratti (Rome, 1953), n" 82; J. INAN et E. ROSENBAUM, Roman and Early Byzantine portrais sculpture in Asia Minor (Londres. 1966), n' 12. pl. 8: K. FITTSCHEN et P. ZANKER. Katalog der römischen Porträts in den Capitolinischen Museen und den anderen kommunalen Sammlungen der Stadt Rom, III (Mayence. 1983), n" 57. pl. 73-75. 
ou contemporäins de leurs règnes $\left({ }^{8}\right)$. J. Ch. Balty a cru pouvoir reconnaître dans la tête de Mariemont un portrait de Lucilla, l'épouse de Lucius Vérus $\left({ }^{9}\right)$. On ne le suivra pas dans cette tentative d'identification. En effet, les maigres indices dont on dispose ne sont pas suffisants pour l'appuyer : le visage assez plein ne constitue pas, à lui seul, un élément probant; par ailleurs, certains traits, comme, par exemple, la forme du front, conviennent mal à Lucilla (fig. 4 et 9), et moins encore à d'autres impératrices, telle Crispina $\left({ }^{10}\right)$.

Il est frappant que le portrait n'ait pas de pupilles incisées, alors que cet usage apparaît dès l'époque d'Hadrien et $s$ 'impose rapidement dans tout l'Empire, les exceptions étant surtout localisées en Orient. Dès lors, une datation avant le milieu du $2^{\mathbf{e}}$ siècle après J.-C. est vraisemblable. Du point de vue du style, l'emploi du ciseau pour rendre les volumes, le refus des effets d'ombre obtenus en creusant de profonds sillons à l'aide du foret indiquent plutôt le début de l'époque impériale $\left({ }^{11}\right)$. La couronne de cheveux, qui est la partie la mieux détaillée, devrait permettre aussi la datation la plus sûre : son aspect irrégulier, presque floconneux semble tout à fait imaginable à la fin de l'époque flavienne ou même sous Hadrien. Un chignon de ce type a pu parfaitement être porté par des jeunes filles dès la fin du règne d'Hadrien avant de

(") Cf. p. ex., K. Fittschen, Die Bildnistypen der Faustina minor und die Fecunditas Augustae, dans Abhundlungen der Akademie zu Göttingen, Philologisch-historische Klasse, 3. Folge, n" 126 (Göttingen, 1982), en particulier le type 1 de Faustine et les types 1 et 2 de Lucilla. Cf. FITTSCHEN et ZANKKR, op. cit., $n^{\prime \prime 2}$ 23. pl. 32 (Faustine la Jeune, type 9, chignon en bosse). n" 115 , pl. 145 et suiv.: $n^{\prime \mathrm{s}} 118$ et suiv.. pl. 149-151, ainsi que le $n^{\prime \prime} 125$, pl. 157; K. Kilge et K. LfHMANN-HaRTLEBH. Die antiken GroßBhronzen. III (Berlin et Leipzig, 1927), pl. 10): A. Guliano, Catalogo dei ritratti Romani del Museo Profano Lateranense (Rome, [957). $\mathrm{n}^{\prime \prime}$ 70, pl. 43. - Malheureusement, dans la plupart des cas, le revers des portraits n'est pas reproduit.

(") Ci-dessus n. 3. M. Whintr, Boreas, 3, 1980\%, p. 70, et FITTSCHEN. op. cit..p. 69 et suiv., ne la mentionnent pas.

(ii) Pour Lucilla, of. M. WhGNHR, Die Antoninen (Berlin. 1939), p. 74 et suiv. . et Boreas, 3, 1980, p. 67 et suiv.: Fitrs(HFN. op. cit.. p. 69-81. pl. 44-48 (pour la chevelure ondée, of. won type 2) : les yeux de Lucilla sont plus allongés, ses paupieres plus larges, son front est plus bombé. sa bouche encore plus petite etc. Pour Crispina. voir Weckek, op. cit., p. 74 et suiv. et 98 et suiv.; devenir un élément assez fréquent de la coiffure officielle des impératrices au temps de Faustine la Jeune et de Lucilla.

L'aspect flou de la couronne de cheveux n'est pas sans rappeler des images de déesses du classicisme tardif et, à y regarder de plus près, sa disposition évoque des ouvres de la fin du $5^{\mathrm{e}}$ siècle avant J.-C. On remarque sur la tempe gauche un motif de mèches entrecroisées. Ce motif se retrouve à la même place sur la tête de l'Amazone dite "de Sosiklès" $\left({ }^{12}\right)$, type que l'on peut attribuer avec une quasi certitude à Polyclète (fig. 5, 6 et 10) et dont la tête se distingue au premier regard de celle du type Lansdowne-Sciarra (BerlinCopenhague) attribuable à Crésilas. La coïncidence pourrait être fortuite, car l'impression générale et le rendu des détails ne correspondent pas à ce qu'on attendrait d'une statue du haut classicisme ou d'une réplique romaine d'une telle statue. En outre, l'arrière de la tête de l'Amazone a un profil beaucoup plus allongé et sa chevelure est nouée tout à fait autrement sur la nuque.

Néanmoins, une comparaison plus poussée entraîne la constatation étonnante que ce motif n'est pas une coïncidence isolée et que, au contraire, d'autres traits de la chevelure de l'Amazone se retrouvent sur la
FITTSCHEN, op. cit., p. 82-88, pl. 59-66 : ses yeux sont plus allongés. la paupière supérieure est plus large. la chevelure structurée autrement.

(11) Par contre, l'emploi stylistique du foret dans la chevelure, de même que l'incision des pupilles n'étaient pas encore généralisés en Orient à l'époque antonine ( seule la provenance supposée de la tête de Mariemont s'oppose à I'hypothèse d"une provenance orientale).

(12) Voir H. Von Steuben. Polyklet (Tübingen. 1974). pl. 48, cf. les détails aux pl. 49-5 I; W. HHI BIfi, Führer durch dite äffentichen Sammlungen in Rom. $4^{4}$ éd., II (Tübingen, 1966). n" 1592. - Sur l'A mazone d'Éphèse, voir M. WFBR: Jahrbuch des deutschen archäologischen Instituts, 91. 1976, p. 56 et suiv. et 93. 1978. p. 175-183; T. DoHR, ibid., 94, 1979, p. 125 et suiv, (avec bibliographie); en dernier lieu, E. HARRISON dans The eye of Greece (Cambridge, 1982), p. 65 et suiv.. pl. 17 et suiv. (dont on ne retiendra pas la répartition des types; ses datations tardives sont notamment contredites par les pl. 20-21). La couronne de cheveux se distingue plus nettement sur les types Herculanum-Tivoli et l.ansdowne-Sciarra (voir, p. ex, ibid. pl. 17 et 22). 


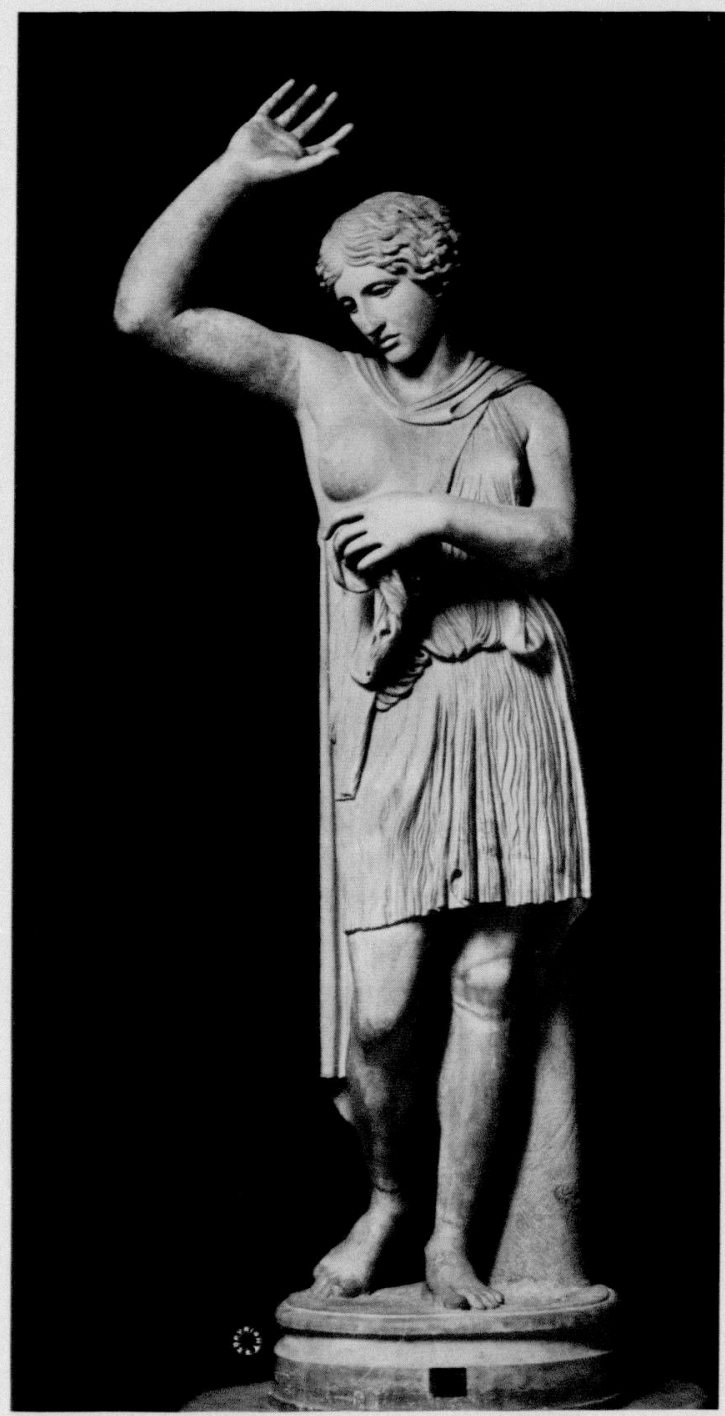

Fig. 6 : Amazone de Sosiklès

Rome. Musée du Capitole (Photo Anderson)

tête de Mariemont. Simplement, l'imitation n'est pas servile, mais se contente de respecter à peu près l'ordonnance générale du modèle. Les proportions, la structure, l'articulation des détails diffèrent. Au lieu de la division continue des ondulations en une superposi- tion de mèches séparées selon la technique du bronze. le portrait présente des incisions plus courtes et irrégulières, qui s'insinuent à l'intérieur des mèches, s'arrêtent au milieu d'une boucle pour reprendre plus loin et donnent une impression plus vivante, plus floconneuse.

Outre l'usure de l'épiderme. ce traitement rend difficile de distinguer et de suivre les différentes boucles. Les trois ou quatre mèches qui surmontent le front offrent pourtant des analogies indéniables. Alors que, chez l'Amazone, les bandeaux recouvrent tout le crâne jusqu'à l'arrière, ici, un trait ondulé sépare la couronne de cheveux du sommet de la tête es se détache harmonieusement sur le ruban. Vers l'arrière, le rendu est quelque peu déformé et simplifié dans le sens d'un mouvement plus naturel de la chevelure, mais ce n'est qu'au voisinage du chignon qu'un changement radical intervient afin d assurer une transition correcte vers les mèches qui vont former la natte. C'est pourquoi aussi les cheveux sont relevés sur la nuque et qu'on ne retrouve plus les petits bouts effilés, typiquement polyclétéens, qui, chez l'Amazone, sortent de l'élégant enchevêtrement des mèches horizontales.

Un dessin schématique illustrerait au mieux la comparaison. On pourrait ainsi, par exemple, suivre les trois mèches du bandeau de droite : si une partie de la mèche inférieure de l'Amazone n'apparait sous la suivante qu'après la première ondulation, dans le portrait, la totalité de la même mèche sort exactement au même endroit de dessous sa voisine pour la croiser ensuite en formant le motif significatif que j'ai déjà signalé. Au-dessus de ce motif, la troisième boucle de l'Amazone disparaît sous les deux autres, qui surgissent juste au-dessus d'elle; sur le portrait, ces trois boucles sont distinguées par des sillons tellement discrets qu'on doit les considérer comme formant une seule grosse mèche à peine légèrement tordue sur ellemême, qui n'existe évidement pas chez l'Amazone. Il en va de même un peu plus loin, au-dessus de l'oreille gauche : entre les deux mèches de l'Amazone en surgit une troisième qui les recouvre immédiatement; sur le portrait, toutes trois ne forment plus qu'une seule masse confuse. Pourtant, prise séparément chaque mèche de l'Amazone trouve son pendant sur le portrait. 


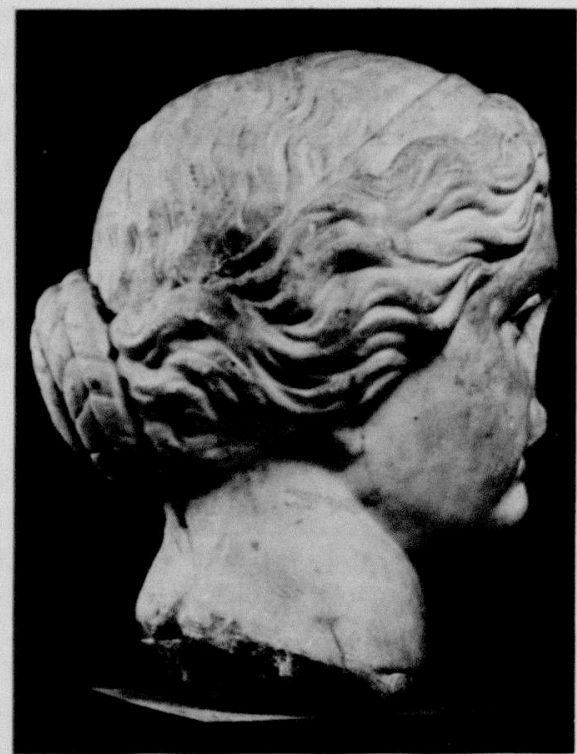

Fig. 7 : Portrait de femme, profil droit Musée royal de Mariemont (Photo du Musée)

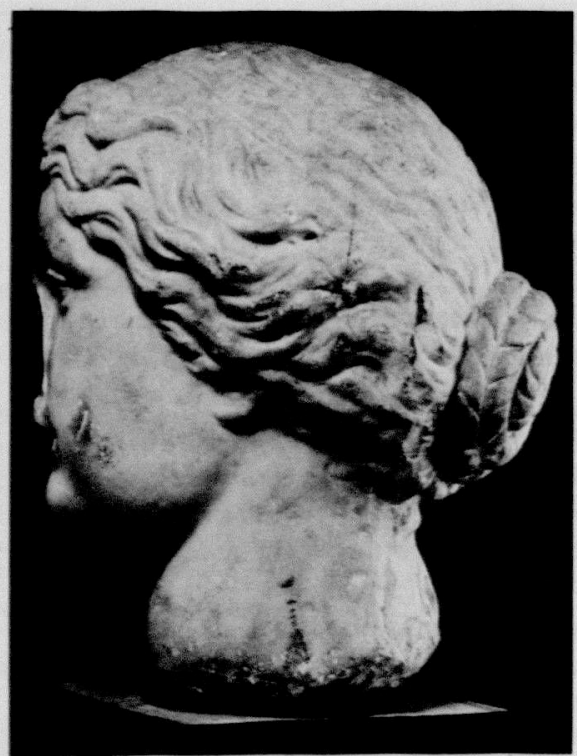

Fig. 8 : Portrait de femme, profil gauche Musée royal de Mariemont (Photo du Musée)

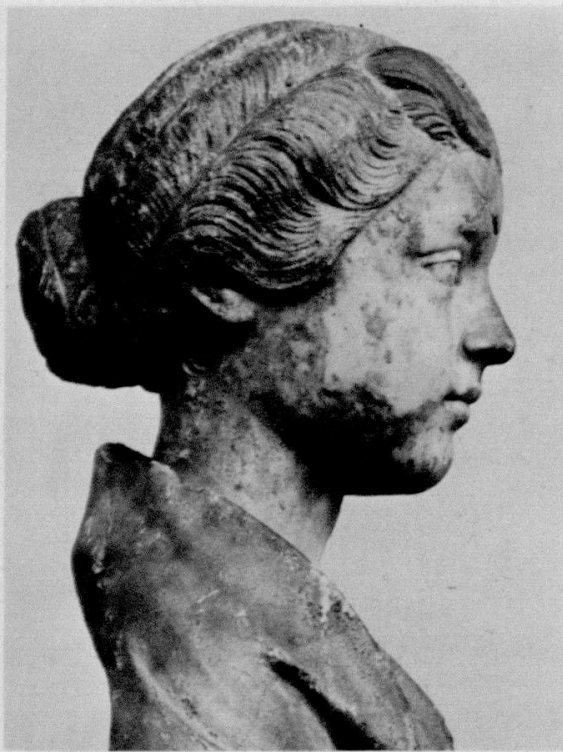

Fig. 9 : Portrait de Lucilla, profil droit Londres, British Museum, Inv. 1912 (Photo British Museum)

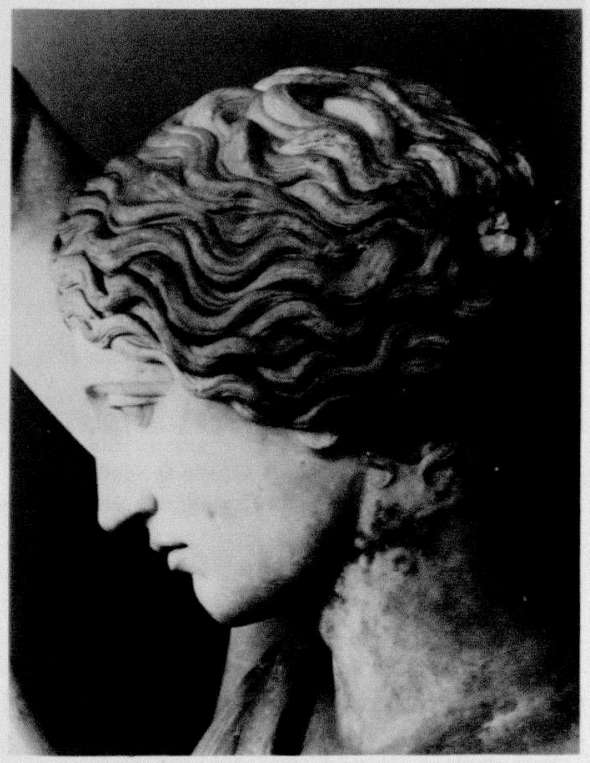

Fig. 10 : Tête de l'Amazone de Sosiklès, profil gauche Rome. Musée du Capitole

(Photo Deutsches archäologisches Institut, Rome) 


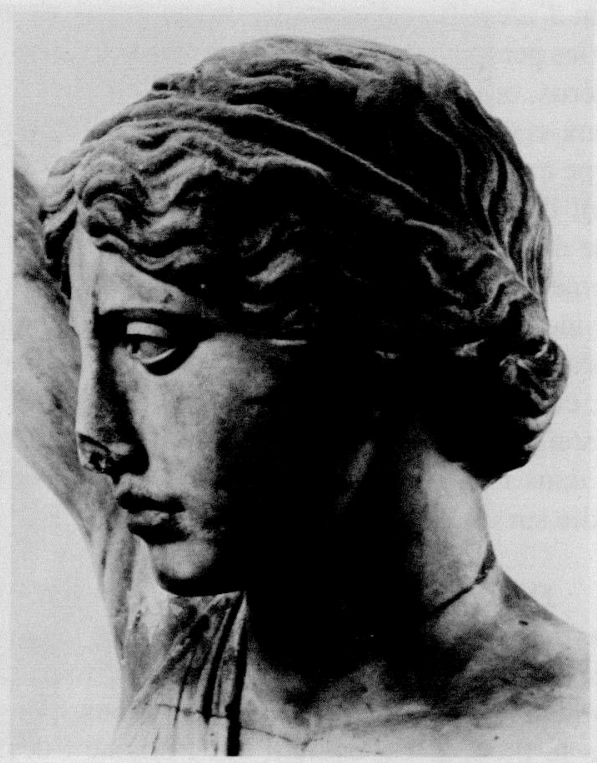

Fig. 11 : Tête d'Amazone, profil gauche Petworth House

(Photo Forschungsarchiv für römische Plastik,

Köln. By kind permission of the National Trust)

Sur le côté gauche de celui-ci, les ondulations sont fortement accentuées. Ici, la forme des boucles rappelle celles, par exemple, de Faustine la Jeune, sans pour autant atteindre leur habituelle symétrie ni leur exécution sèche, le plus souvent recreusée au foret. II en résulte une nette simplification du "désordre systématique" de la structure des boucles polyclétéennes, qui entraîne le sacrifice de boucles entières, comme les

(13) La tête de Petworth House (fig. 11) porte au méme endroit un bandeau sur lequel la chevelure repose dans la nuque HARRISON, art. cité, pl. 20, c-d.; WEBER, art. cité 1976, p. 65 , fig. 28-29; H. OEHL.ER, Foto + Skulptur. Catalogue (Cologne, 1980), $\mathrm{n}^{\circ} 80, \mathrm{pl} .4-7$. Cette tête est une variante simplifiée de la tête du type Lansdowne-Sciarra, dont l'attribution proposée par WFBER, art. cité 1978 , p. 175 et suiv., et HARRISON, art. cité, p. 75, au torse Mattei apparaît douteuse d'après les photographies. Elle est également assez proche de la tête de Mariemont par sa maniere d'adapter le modele et le rendu de la chevelure. Je tiens a remercier ici $\mathrm{H}$. Wrede, qui a attiré mon attention sur les traces de remaniement du ruban de la tête de Mariemont. Mais contrairement à la tête de Münster (cf. n. I) et à une tête féminine provenant du caveau frisons devant les lobes des oreilles. C'est ainsi que, chez l'Amazone, les deux boucles supérieures de la couronne au-dessus du front sont disposées plus ou moins symétriquement et une troisième mèche, en partie perpendiculaire, les recoupe dans le bas, alors que, dans le portrait, les trois mèches deviennent parallèles et se chevauchent presque sans contraste. Devant l'oreille - comme dans la coiffure de Faustine - un creux supplémentaire, large et profond, souligne la convexité supérieure des ondulations, tandis qu'ici les boucles de l'Amazone sont relevées. Plus loin, ces boucles recoupent, dans le portrait, celles qui se trouvent au-dessus et qui, chez l'Amazone, partent trop haut pour pouvoir conduire au chignon.

Il reste à éclaircir l'origine et la signification du ruban $\left({ }^{13}\right)$ dans les cheveux, qui n'est pas repris au type de l'Amazone de Polyclète et n'appartient pas davantage à la coiffure romaine. Un tel lien ne se trouve pas, en tout cas, normalement dans les portraits du $2^{\mathrm{e}}$ siècle après J. - C. En outre, les bouts plats qui pendent dans la nuque, et qui ne sont guère perceptibles de profil, semblent témoigner d'un remaniement.

La tête de l'Amazone était tournée vers la droite exactement comme celle du portrait, mais celleci l'est davantage et s'incline plus fortement en avant, ce qui s'accorde avec les plis nettement visibles sur la partie droite du cou. La coupure du cou de cette tête, destinée à être encastrée, indique qu'elle appartenait à une statue habillée. Étant donné le mélange de traits empruntés à un modèle idéal et au portrait, on pourrait penser aussi bien à une statue drapée de type courant qu'à la reprise du type de l'Amazone $\left({ }^{14}\right)$. Certes, au

funéraire de Cologne-Weiden (F. FREMERSDORF, Das Römergrah von Weiden bei Köln (Cologne, 1957), p. 38-39, pl. 26-31; H. JUCKł:R, Das Bildnis im Blätterkelch (Olten e.a., 1961), p. 89-90, n" St 35, pl. 34), que H. Wrede m'a également signalée, ce portrait $n^{\circ}$ a visiblement pas subi de remaniements importants à une époque postérieure de l'antiquité

$\left({ }^{14}\right)$ Hel. BIG, op. cit., n" 1393; von SthUBEN, op. cit. p. 56 et suiv., pl. 40-43. La tête-portrait de la réplique du Musée du Capitole (Salone 19: von Struben, op. cit., p. 45; H.S. Jonfs, The sculptures of the Museo Capitolino (Oxford, 1912), p. 286. pl. 69) n'appartient pas au corps, auquel elle est liée par une fixation moderne au plâtre : elle ne constitue donc pas un parallèle pour notre portrait. 
contraire des authentiques Amazones d'Éphèse, celle de Sosiclès porte un manteau sur les épaules, ce qui lui permettrait, en théorie, de recevoir une tête encastrée. Toutefois, cela n' aurait pas grand sens puisque la chair nue reparait en-dessous : que la tête ait été exécutée à part ne saurait donc s"expliquer au mieux, dans cette hypothèse, que si elle l'a été par un autre sculpteur que le reste de la statue. Une reproduction exacte du modèle eût d'ailleurs exigé que le décolleté descende plus bas à l'arrière et moins bas du côté droit, là où se trouve la cassure. Dès lors, à côté de la simple statue drapée. il ne reste que la possibilité d'une adaptation libre du type de l'Amazone, qu'auraient explicité, par exemple. des éléments ou accessoires caractéristiques, un vêtement particulier, voire une cuirasse. Ceci soulève la question de la signification iconographique de la statue.

Nous n'avons donc affaire ici ni à une copie pure et simple, ni à une copie-portrait. Le mieux est de la considérer comme un portrait, dans lequel on a intégré les éléments les plus caractéristiques d'une cuvre idéale déterminée, facilement identifiable à l'époque par les connaisseurs de la sculpture antique. Il est même possible que d'autres éléments, aujourd'hui perdus, rendaient cette identification encore plus claire.

De telles confusions des genres sont très fréquentes dans les portraits romains. De mème que les modèles varient tant au point de vue du contenu qu'à celui du style et témoignent de choix qui ne correspondent nullement au goût classicisant pour les chefsd'ouvre reconnus, de même il existe des degrés très différents de mélange des deux sphères. Choix du modèle et degré du mélange dépendent de la destination et de la fonction des ouvres, ainsi que des intentions de ceux qui les font ériger. En outre, la relation entre l'artiste ou son client et les modèles idéaux évolue avec le temps. Une bonne partie de ces "idéalisations" " "inspirent d'images divines : il s"agit essentiel-

(15) PETRowe. Santiom. 29. C. H. WREDE. Comseratio in formum deorum. Vergötlichte Privatpersonen in der rimischen Kaiserzeit (Mayence. 1981). p. 67-68. Sur lensemble du phenomène. cf. en général. WRtDE. op. ait. p. 73 et suiv.. 94 et suiv., lement d'identifier, d'assimiler ou, au moins, de comparer les personnages représentés à des dieux, parfois à des héros, ou encore de les présenter comme des reflets de ceux-ci (quand le modèle est un héros, la référence à un type exemplaire devait d'ailleurs être primordiale). Le but du procédé n'est pas de reproduire pour euxmême des chefs-d'œuvre renommés; ces derniers sont seulement le meilleur moyen d'obtenir des représentations idéales sur mesure. Le phénomène se manifeste tant dans la statuaire honorifique que dans l'art funéraire, et naturellement aussi dans les autres genres : que l'on songe, par exemple, au portrait de lui en Hermès que. dans le roman de Pétrone, Trimalcion avait fait peindre sur un mur de sa maison $\left({ }^{15}\right)$.

Dans la plupart des cas, il s'agit réellement d"une déification - explicite ou implicite. Une bonne partie des statues honorifiques d'époque romaine est constituée par des portraits de souverains, pour lesquels le procédé est courant dès l'origine : déjà, les souverains hellénistiques se faisaient représenter volontiers en Héraclès, en Dionysos, en Apollon etc. L'empereur romain se fait représenter de préférence en Jupiter: $d$ 'autres identifications, comme, par exemple. celle des impératrices à Junon ou à Cérès, en découlent

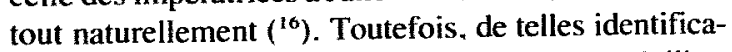
tions n'ont jamais été considérées comme un privilège impérial : tout au long de l'Empire, à l'instar des portraits des empereurs, des portraits privés de plus en plus nombreux emprunteront des traits divins; d'abord attestés surtout à Rome et dans les environs immédiats, ils connaîtront une large diffusion à partir du $2^{e}$ siècle après J.C.C.

En ce qui concerne les statues honorifiques (ou les statues que le propriétaire se fait ériger dans sa maison), il est possible que des membres de l'aristocratie aient été représentés ainsi, bien que les documents sûrs soient particulièrement rares et proviennent surtout d'Orient $\left({ }^{17}\right)$. En revanche. les statues funéraires. de même d'ailleurs que les reliefs, représentent le

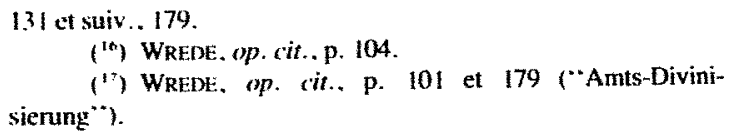




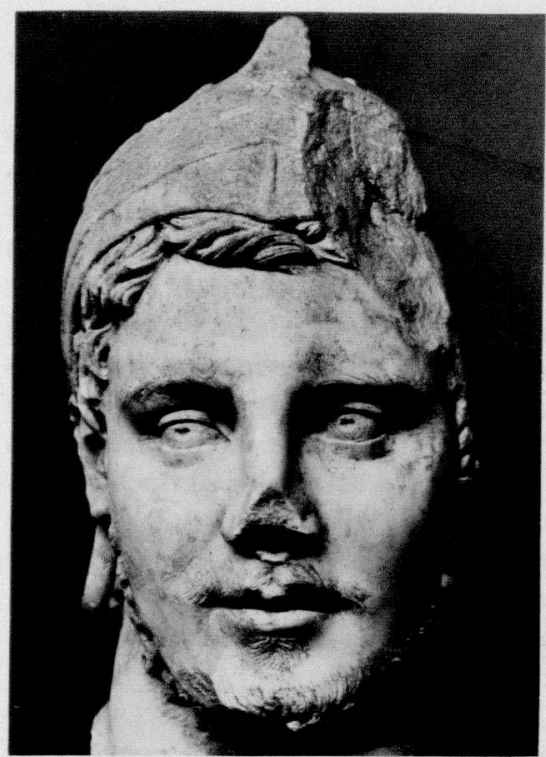

Fig. 12 : Portrait d'homme en Mars Borghèse Rome, Musée national des Thermes

(Photo Deutsches archäologisches Institut, Rome)

plus souvent des personnes dont on peut difficilement supposer qu'on en a fait d'autres portraits. Dans la plupart des cas, ces représentations doivent s'expliquer par le phénomène de l" "apothéose privée", que Wrede a étudié de manière exhaustive et qu'il fait remonter principalement aux affranchis qui bénéficièrent d'une promotion sociale au $1^{\mathrm{er}}$ et au début du $2^{\mathrm{e}}$ siècle après J.-C. $\left({ }^{18}\right)$. Mais, même dans ces cas, on peut parfois n'avoir eu en vue qu'une simple comparaison ou association avec la divinité; ceci doit être encore plus souvent et plus nettement vrai lorsque le modèle est l'image d'un héros. Il n'est cependant pas possible de distinguer les conceptions divergentes dans chaque cas particulier : souvent d'ailleurs, la représentation figurée, volontairement ambiguë, ne cherche pas à imposer au spectateur un choix entre les interprétations possibles.

(18) WrEDE, op. cit., p. 94 et suiv., 160 et suiv.

( $\left.{ }^{19}\right)$ Voir, en particulier, WREDE, op. cit., p. 75-79, 98-99, 133-138; cf. p. 195 et suiv. son catalogue par divinités. On comparera tout spécialement à la tête de Mariemont les portraits de femmes

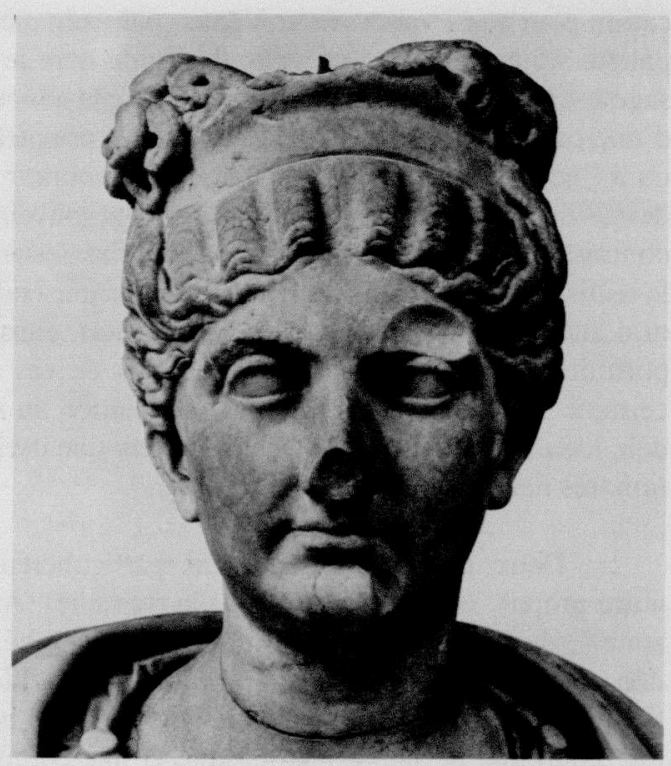

Fig. 13 : Portrait de femme coiffée comme Vénus Woburn Abbey

(Photo Forschungsarchiv für römische Plastik, Köln. By kind permission of the Marquess of

Tavistock, and the Trustees of the Bedford Estates)

Je n'ai pas l'intention ici de brosser un tableau d'ensemble du phénomène ni d'en faire une étude systématique. Quelques remarques suffiront $\left({ }^{19}\right)$. Pour les femmes, l'assimilation la plus fréquente est avec Aphrodite; on en trouve également avec Diane (pour les jeunes filles), plus souvent avec Cérès, parfois avec Fortuna. Pour les hommes, Hermès-Mercure domine les représentations privées; on rencontre également Hercule, ainsi que Liber-Dionysos. Mars, qui n'apparaît que sporadiquement, prend surtout l'aspect de l'Arès Borghèse (fig. 12) associé, dans les portraits de couples, à une Vénus du type de l'Aphrodite de Capoue. De même que la façon de rendre l'assimilation peut aller de l'emprunt de quelques éléments ou attributs à l'imposition d'une tête-portrait sur un type connu ou encore se limiter à une allusion à un modèle idéal dans les traits du visage, l'intensité de l'identifi-

en Vénus, où l'association avec la divinité n'est suggérée que par le nœud de cheveux au sommet du crâne : cf. WREDE, op. cit., $\mathrm{n}^{\circ} 295$ et suiv., pl. 37. Sur le groupe Vénus-Mars, voir FelletI MAJ, op. cit.. $\mathrm{n}^{\circ} 236$. 
cation peut aussi varier. Pour Vénus, par exemple, la palette s'étend d'une Aphrodite de Cnide dont le visage est remplacé par un portrait à la simple adoption d'une coiffure inspirée de celle du modèle praxitélien ou à l'adjonction d'un nœud de boucles décoratif audessus du front (fig. 13). Ces statues offrent souvent un contraste brutal entre la beauté du corps classicisant et le réalisme du visage qui ne fait grâce d'aucune laideur ni d'aucun stigmate de l'âge. Souvent aussi, dans les portraits de femmes, on remarque dans ce cas une certaine idéalisation, une certaine adaptation au modèle idéal. À cet égard, on peut constater une oscillation très nette au cours des temps.

Deux exemples intéressent particulièrement notre propos, car ils tombent sous la rubrique "Amazone", du moins au sens d'Amazone, de Tyché, de déesse de la cité : d'abord, un portrait de jeune fille de l'époque de Claude conservé au Vatican et qui porte une couronne de laurier, un casque et une couronne tourelée; ensuite, un portrait privé d'époque flavienne, autrefois à Velletri, qui porte un casque $\left({ }^{20}\right)$. Bien qu'on puisse penser à une Minerve ou à une autre déesse guerrière, le processus d'identification est ici certainement différent. Une déesse de la cité suppose une relation directe avec une cité déterminée : il pourrait s'agir, par exemple, d'une statue élevée par cette cité à un membre d'une famille de bienfaiteurs ou de la famille impériale. Le procédé s'apparente à l'emprunt déjà signalé d'un élément de coiffure généralement associé à Vénus.

Dans le cas qui nous occupe, il n'est pas question de déification, puisque les Amazones d'Ephèse ne sont pas des déesses : au contraire, on les montre grièvement blessées au combat ! Étant donné que, dans

("1) WREDE. $o p$. cit., p. 74 et 304-305, n" 290, pl. 36,2-5 (suppose qu"il pourrait s"agir de Claudia. la sour de Néron): p. 74 et 283. $\mathrm{n}^{\prime 2} 234$ (inédite. pas sùrement antique).

(2) D'autres héros sont particulièrement cités comme modèle moraux dans les discours et poèmes funéraires. comme. p. ex.. Thésée ou Achille : (voir Wrent; p. 51-53). Les jeunes femmes, en revanche, sont comparées moins souvent ả des herrönes qua à de belles déesses. Pour une comparaison avec une Amazone sur le plan de la beauté, voir W. Ptik. Griechische Vers-Inschriften (Berlin. 1955), $\mathrm{n}^{*} 1164=$ du meme. Griechische Grahgedichte griechivch les autres exemples que nous connaissons, la réfẻrence n'est pas au modèle sculpté mais à ce qu'il représente, on ne cherchera pas ici l'imitation du style de Polyclète (dans ce cas', on s'attendrait à une copie plus fidèle). Dès lors, l'intention de l'artiste ne saurait avoir été que de magnifier et d'idéaliser quelqu'un de façon générale sous la forme classique d'une héroïne. Celle-ci fait fonction de modèle moral, un modèle qu'il est facile de transposer d'emblée et, pour ainsi dire, tout naturellement dans la présentation figurée d'une personne déterminée. Le choix de ce modèle avait-il un rapport avec le caractère de la personne représentée? Dans l'affirmative - et pour autant que nous le sachions il pourrait faire allusion à sa beauté juvénile ou encore à son courage, peut-être à son amour conjugal, par référence à celui de l'Amazone Antiope pour Thésée. mais certainement pas à son engagement en faveur de l'émancipation féminine $\left({ }^{21}\right)$ !

Les portraits romains en marbre trouvés dans les provinces frontalières du nord-ouest de l'Empire sont relativement rares, si on les compare à la masse de ceux qui proviennent du bassin méditerranéen. De plus, ils sont surtout concentrés dans les grandes villes, en particulier dans les chefs-lieux de provinces et de cités. On connaît pourtant quelques exemples de portraits privés de haute qualité, qui ont été trouvés à la campagne, notamment dans des villas appartenant à de riches Romains - tel celui de Lullingstone (Kent) (22) - et qui en représentent certainement les propriétaires. On pourrait donc considérer la tête de Mariemont comme le portrait de l'épouse du propriétaire d'une villa romaine des environs de Maastricht, qui aurait voulu se faire représenter aux yeux de sa familia et de ses hôtes sous une forme transfigurée par des allusions à l'Amazone de Polyclète.

und deutsch (Berlin, 1960). n" 392 (Insiptiones Graecae. XIV. $\mathrm{n}^{\prime \prime}$ 1839); H. BRANDENBLIRG, Jahrbuch des deutschen archïologisshen Instituts, 82, 1967, p. 243 et n. 141, insiste également sur le fait que même les comparaisons avec Endymion. Hylas ou Alceste visent en premier lieu la beauté du modèle mythique. Sur les représentations d"Amazones dans l'art antique, cf. aussi A. BAMmER, Hephaistes. 4. 1982. p. 67-77. qu'on ne suivra pas sur tous les points.

(2) Voir G. W. MEATES, Lullingstone Roman Villa (Londres e.a., 1955), p. 70 et suiv., tig. 17-29. 
Pourtant, qu'une tête, censément trouvée au tournant du siècle, ait été aussi fortement restaurée et que, dans la foulée, on ait encore trouvé moyen de la débarrasser de ces restaurations éveille quelques soupçons. Ajoutons-y le trou de fixation (?) grossièrement creusé à l'arrière. On peut donc envisager la possibilité que la tête soit restée un certain temps dans une collection privée et qu'elle ait peut-être passé par le commerce des antiquités. Dès lors, des doutes se font jour quant au lieu de sa découverte. Celui-ci ne peut être mis en relation avec aucune ruine connue ni aucune fouille ancienne. Des informations aussi vagues appellent d'office le scepticisme, même si l'on n'a pas le droit de les rejeter a priori $\left({ }^{23}\right)$. Si done, comme on l'a dit, il est tout à fait possible que la pièce ait été effectivement trouvée dans les provinces du nordouest, une provenance méridionale - par exemple, comme souvenir d'un voyage à Rome - n'est pas exclue. Mais, à ce sujet, on ne peut que se livrer à des conjectures.

(Traduit de l'allemand par G. Donnay) 